\title{
A GENERAL QUADRATURE FORMULA USING ZEROS OF SPHERICAL BESSEL FUNCTIONS AS NODES*
}

\author{
Riadh Ben Ghanem ${ }^{1}$ and Clément FrappieR ${ }^{1}$
}

\begin{abstract}
We obtain, for entire functions of exponential type satisfying certain integrability conditions, a quadrature formula using the zeros of spherical Bessel functions as nodes. We deduce from this quadrature formula a result of Olivier and Rahman, which refines itself a formula of Boas.

Résumé. Nous obtenons, pour les fonctions entières de type exponentiel satisfaisant certaines conditions d'intégrabilité, une formule de quadrature utilisant les zéros des fonctions de Bessel sphériques comme nœuds. Nous déduisons de cette formule un résultat de Olivier et Rahman, lequel est lui-même un raffinement d'une formule préalablement obtenue par Boas.
\end{abstract}

AMS Subject Classification. 30D10, 33C10, 41A55, 65D32.

Received: July 13, 1998.

\section{INTRODUCTION} that

If $f$ is an entire function of exponential type $2 \pi$ belonging to $L^{1}(-\infty, \infty)$ then it was proved by Boas [3]

$$
\int_{-\infty}^{\infty} f(x) \mathrm{d} x=\sum_{k=-\infty}^{\infty} f(k)
$$

This formula was improved by Olivier and Rahman [8] as follows (see also [5] and [7])

Theorem A. Let $m$ be a non-negative integer. For every entire function $f$ of exponential type $\sigma<2(m+1) \pi$ such that $f(x)$ is integrable in the sense of Cauchy on $(-\infty, \infty)$, we have

$$
\int_{\rightarrow-\infty}^{\rightarrow \infty} f(x) \mathrm{d} x=\sum_{\mu=0}^{m} c_{m, 2 \mu} \sum_{k=-\infty}^{\infty} f^{(2 \mu)}(k)
$$

Keywords and phrases. Quadrature, Bessel functions, Cauchy, residue.

* The research of the second author was supported by the Natural Sciences and Engineering Research Council of Canada Grant No. OGP 000 9331. The first author acknowledge a partial support from the same grant.

${ }^{1}$ Département de Mathématiques et de Génie Industriel, École Polytechnique, CP 6079, Succ. Centre-Ville, Montréal, Québec, Canada H3C 3A7. e-mail: clement.frappier@courrier.polymtl.ca 
where $c_{0,0}=1$ and the constants $c_{m, 2 \mu}$ are given, for $m \geq 1$ and $0 \leq \mu \leq m$, by

$$
\prod_{j=1}^{m}\left(1+\left(\frac{x}{2 \pi j}\right)^{2}\right)=\sum_{\mu=0}^{m} c_{m, 2 \mu} x^{2 \mu} .
$$

It may be recalled that

$$
\int_{0}^{\rightarrow \infty} f(x) \mathrm{d} x
$$

is said to exist in the sense of Cauchy on $(0, \infty)$ if $f$ is integrable on $(0, X)$ for every $X$ and $\lim _{X \rightarrow \infty} \int_{0}^{X} f(x) \mathrm{d} x$ exists. Similarly, we define the integrability in the sense of Cauchy on $(-\infty, 0)$. We say that $f$ is integrable in the sense of Cauchy on $(-\infty, \infty)$ whenever it is integrable in the sense of Cauchy on both intervals $(0, \infty)$, $(-\infty, 0)$ and we denote the integral by

$$
\int_{\rightarrow-\infty}^{\rightarrow \infty} f(x) \mathrm{d} x
$$

If $f$ is Lebesgue integrable on $(-\infty, \infty)$ then it is integrable in the sense of Cauchy on $(-\infty, \infty)$ and

$$
\int_{\rightarrow-\infty}^{\rightarrow \infty} f(x) \mathrm{d} x=\int_{-\infty}^{\infty} f(x) \mathrm{d} x .
$$

Let $J_{\alpha}(z)$ be the Bessel function of the first kind of order $\alpha$. The function

$$
G_{\alpha}(z):=\frac{J_{\alpha}(z)}{z^{\alpha}}=\sum_{k=0}^{\infty}(-1)^{k} \frac{z^{2 k}}{2^{\alpha+2 k} k ! \Gamma(k+\alpha+1)}
$$

is entire and of exponential type 1 . We denote by $j_{k}=j_{k}(\alpha)$ the zeros of $G_{\alpha}(z)$, which are simple whenever $\alpha$ is not a negative integer, ordered such that $0<\left|j_{1}\right| \leq\left|j_{2}\right| \leq \ldots$ and $j_{-k}=-j_{k}$ for $k=1,2, \ldots$

Since the nodes used in the quadrature formula of Theorem A are the point zero and the roots of the function

$$
G_{\frac{1}{2}}(z)=\sqrt{\frac{2}{\pi}} \frac{\sin z}{z}
$$

it is natural to wonder if there exists a generalization of Theorem A using the zeros of Bessel functions of order $n+1 / 2$ for every integer $n$. The Bessel functions of order $n+1 / 2, n=0, \pm 1, \pm 2, \ldots$, are known as the spherical Bessel functions.

From now on we will often write $\alpha$ instead of $n+1 / 2, n=0, \pm 1, \pm 2, \ldots$, and $j_{k}=j_{k}(n+1 / 2)$ denote the zeros of $G_{\alpha}(z)$.

\section{Statement of the Results}

Our main result is a generalisation of (2).

Theorem 1. Let $m$ be a non-negative integer and let $n$ and $p$ be two integers such that

$$
p \geq \begin{cases}(2 n+1)(2 m+1) & \text { if } n \geq 0 \\ 2 n+1 & \text { if } n<0\end{cases}
$$


For every entire function $z^{p} f(z)$ of exponential type $\sigma<2(m+1) \tau$ such that $x^{p} f(x)$ is integrable in the sense of Cauchy on $(-\infty, \infty)$, we have

$$
\int_{\rightarrow-\infty}^{\rightarrow \infty} x^{p} f(x) \mathrm{d} x=\frac{(-1)^{n} \pi(2 m+1) !}{2^{2 m}(m !)^{2} \tau^{p+1}} \sum_{\substack{k=-\infty \\
k \neq 0}}^{\infty} \sum_{\mu=0}^{m} \sum_{\nu=0}^{2 \mu} \frac{\left(\begin{array}{c}
m \\
\mu
\end{array}\right)\left(\begin{array}{c}
2 \mu \\
\nu
\end{array}\right)}{(2 \mu+1) ! \tau^{\nu}} a_{\mu, \nu}\left(j_{k}\right) f^{(\nu)}\left(\frac{j_{k}}{\tau}\right)
$$

where

$$
a_{\mu, \nu}\left(j_{k}\right):=\lim _{z \rightarrow j_{k}}\left(z^{p}\left(J_{-\alpha}(z) \frac{\left(z-j_{k}\right)}{J_{\alpha}(z)}\right)^{2 \mu+1}\right)^{(2 \mu-\nu)} .
$$

Here $\alpha$ is of the form $n+1 / 2$ and the numbers $j_{k}$ are zeros of the Bessel function of order $\alpha$ arranged as described above.

Remark. In order to formulate more explicitly the quantities $a_{\mu, \nu}\left(j_{k}\right)$, we apply two times the Leibniz's theorem to obtain

$$
a_{\mu, \nu}\left(j_{k}\right)=\lim _{z \rightarrow j_{k}} \sum_{r=0}^{2 \mu-\nu} \sum_{s=0}^{r}\left(\begin{array}{c}
2 \mu-\nu \\
r
\end{array}\right)\left(\begin{array}{l}
r \\
s
\end{array}\right)\left(z^{p}\right)^{(s)}\left(J_{-\alpha}^{2 \mu+1}(z)\right)^{(r-s)}\left(\left(\frac{\left(z-j_{k}\right)}{J_{\alpha}(z)}\right)^{2 \mu+1}\right)^{(2 \mu-\nu-r)} .
$$

Next we define, for every integer $n$ and for each non-negative integer $m$, the functions

$$
\begin{aligned}
\phi_{\alpha}(z) & :=i(-1)^{n} \frac{J_{-\alpha}(\tau z)}{J_{\alpha}(\tau z)} \\
\psi_{\alpha, m}(z) & :=\frac{(2 m+1) !}{2^{2 m}(m !)^{2}} \sum_{\mu=0}^{m}\left(\begin{array}{c}
m \\
\mu
\end{array}\right) \frac{(-1)^{\mu}}{2 \mu+1}\left(\phi_{\alpha}(z)\right)^{2 \mu+1} .
\end{aligned}
$$

If the integer $p$ does not satisfy the condition (3) then we need to take into account a residue at zero.

Theorem 1'. Let $m$ be a non-negative integer and let $n$ and $p$ be two integers. For every entire function $z^{p} f(z)$ of exponential type $\sigma<2(m+1) \tau$ such that $x^{p} f(x)$ is integrable in the sense of Cauchy on $(-\infty, \infty)$, we have

$$
\begin{aligned}
\int_{\rightarrow-\infty}^{\rightarrow \infty} x^{p} f(x) \mathrm{d} x= & -\pi i \operatorname{Res}\left(z^{p} \psi_{\alpha, m}(z) f(z) ; z=0\right) \\
& +\frac{(-1)^{n} \pi(2 m+1) !}{2^{2 m}(m !)^{2} \tau^{p+1}} \sum_{\substack{k=-\infty \\
k \neq 0}}^{\infty} \sum_{\mu=0}^{m} \sum_{\nu=0}^{2 \mu} \frac{\left(\begin{array}{c}
m \\
\mu
\end{array}\right)\left(\begin{array}{c}
2 \mu \\
\nu
\end{array}\right)}{(2 \mu+1) ! \tau^{\nu}} a_{\mu, \nu}\left(j_{k}\right) f^{(\nu)}\left(\frac{j_{k}}{\tau}\right) .
\end{aligned}
$$

We will see in Section 3 how Theorem A may be deduced from Theorem 1'.

Theorem 2. Let $m$ be a non-negative integer and let $n$ and $p$ be two integers satisfying (3). Then Formula (4) holds for every entire function $z^{p} f(z)$ of exponential type $2(m+1) \tau$ such that $x^{p} f(x)$ is Lebesgue integrable on $(-\infty, \infty)$.

Theorem 2'. Let $m$ be a non-negative integer and let $n$ and $p$ be two integers. Then Formula (9) holds for every entire function $z^{p} f(z)$ of exponential type $2(m+1) \tau$ such that $x^{p} f(x)$ is Lebesgue integrable on $(-\infty, \infty)$. 


\section{Corollaries}

From Theorem 1', we deduce the following

Corollary 1. The particular case $n=0, p=0$ and $\tau=\pi$ of Theorem 1' gives Theorem A.

Proof. For $n=0$ and $p=0$, we have $\alpha=1 / 2$,

$$
\begin{aligned}
J_{\alpha}(z) & =J_{\frac{1}{2}}(z)=\sqrt{\frac{2}{\pi z}} \sin z, \quad \frac{j_{k}}{\tau}=\frac{j_{k}(1 / 2)}{\tau}=k, \quad k= \pm 1, \pm 2, \ldots, \\
J_{-\alpha}(z) & =J_{-\frac{1}{2}}(z)=\sqrt{\frac{2}{\pi z}} \cos z,
\end{aligned}
$$

so that $\phi_{\alpha}(z)=i \cot (\pi z)$ and

$$
\psi_{\frac{1}{2}, m}(z)=\frac{i(2 m+1) !}{2^{2 m}(m !)^{2}}=\sum_{\mu=0}^{m}\left(\begin{array}{c}
m \\
\mu
\end{array}\right) \frac{1}{2 \mu+1}(\cot (\pi z))^{2 \mu+1} .
$$

So, $\psi_{\frac{1}{2}, m}(z)$ corresponds exactly to the function $-2 K_{m}(z)$ defined in Formula (3.1) of [9].

On the other hand, applying Theorem 1' and the forthcoming Lemma 3, we have

$$
\int_{\rightarrow-\infty}^{\rightarrow \infty} x^{p} f(x) \mathrm{d} x=-\pi i \sum_{k=-\infty}^{\infty} \operatorname{Res}\left(z^{p} f(z) \psi_{\frac{1}{2}, m}(z) ; z=\frac{j_{k}}{\tau}\right)
$$

where $\operatorname{Res}\left(f(z) \psi_{\frac{1}{2}, m}(z) ; z=0\right)$ is included in the sum for $k=0$.

Finally, according to Lemma 2 of [9] we have

$$
-\pi i \operatorname{Res}\left(z^{p} \psi_{\frac{1}{2}, m}(z) f(z) ; z=k\right)=\sum_{\mu=0}^{m} c_{m, 2 \mu} f^{(2 \mu)}(k)
$$

and the result follows.

Applying Theorem 2 to a function of the form $\left(\frac{J_{\alpha}(\tau z)}{(\tau z)^{\alpha}}\right)^{2 m} f(z)$, we obtain the following

Corollary 2. Let $f$ be a function of exponential type $2 \tau$. For all non-negative integer $m$ and $p$ satisfying (3), we have,

$$
\int_{-\infty}^{\infty} x^{p}\left(\frac{J_{\alpha}(x)}{x^{\alpha}}\right)^{2 m} f\left(\frac{x}{\tau}\right) \mathrm{d} x=\frac{2(2 m) !}{\pi^{2 m}(m !)^{2}} \sum_{\substack{k=-\infty \\ k \neq 0}}^{\infty} \frac{j_{k}^{p-2 \alpha m-2 m-1}}{\left(J_{\alpha}^{\prime}\left(j_{k}\right)\right)^{2 m+2}} f\left(\frac{j_{k}}{\tau}\right)
$$

if the integral on the left-hand side of (10) converges.

\section{Lemmas}

Let $H_{\alpha}^{(1)}(z)$ and $H_{\alpha}^{(2)}(z)$ be the Bessel functions of the third kind and of order $\alpha$, defined by

$$
\begin{aligned}
& H_{\alpha}^{(1)}(z)=J_{\alpha}(z)-i(-1)^{n} J_{-\alpha}(z), \\
& H_{\alpha}^{(2)}(z)=J_{\alpha}(z)+i(-1)^{n} J_{-\alpha}(z) .
\end{aligned}
$$


We consider the following auxiliary functions

$$
g_{\alpha}^{(1)}(z):=\frac{H_{\alpha}^{(1)}(\tau z)}{J_{\alpha}(\tau z)}, \quad g_{\alpha}^{(2)}(z):=\frac{H_{\alpha}^{(2)}(\tau z)}{J_{\alpha}(\tau z)}
$$

and

$$
P_{m}(x):=\frac{(2 m+1) !}{(m !)^{2}} \int_{0}^{x} u^{m}(1-u)^{m} \mathrm{~d} u=\sum_{\nu=m+1}^{2 m+1} a_{\nu} x^{\nu}
$$

We set also

$$
\begin{aligned}
& C_{R}:=\{z:|z|=R\}, \quad C_{R}^{+}:=\{z:|z|=R, \Im(z)=\geq 0\}, \\
& C_{R}^{-}:=\{z:|z|=R, \Im(z) \leq 0\} \text { and } R=R_{N}(\alpha):=\frac{1}{\tau}\left(N \pi+\alpha \frac{\pi}{2}+\frac{\pi}{4}\right)
\end{aligned}
$$

where $N$ is a positive integer. We are now ready to state the following results.

Lemma 1. Let $n$ be an integer, $m$ a positive integer and $\beta$ a real number. If $f$ is an entire function of exponential type $\sigma<2(m+1)$ such that $x^{\beta} f(x)$ is integrable in the sense of Cauchy on $(-\infty, \infty)$, then, with $g_{\alpha}^{(1)}$ and $g_{\alpha}^{(2)}$ as in (13), we have

$$
\lim _{N \rightarrow \infty} \int_{C_{R}^{+}} z^{\beta} f(z) P_{m}\left(\frac{g_{\alpha}^{(1)}(z)}{2}\right) \mathrm{d} z=0
$$

and

$$
\lim _{N \rightarrow \infty} \int_{C_{R}^{-}} z^{\beta} f(z) P_{m}\left(\frac{g_{\alpha}^{(2)}(z)}{2}\right) \mathrm{d} z=0
$$

Proof. Note that, by a simple adaptation of the proof, Lemma 5 of [2] is obviously valid without the restriction on the parity of $f(z)$ whenever $x^{\beta} f(x)$ is integrable in the sense of Cauchy on $(-\infty, \infty)$. So, using this Lemma 5 , we obtain

$$
\lim _{N \rightarrow \infty} \int_{C_{R}^{+}} z^{\beta} f(z / \tau)\left(g_{\alpha}^{(1)}(z / \tau)\right)^{\nu} \mathrm{d} z=0
$$

for $\nu \geq m+1$. Thus

$$
\lim _{N \rightarrow \infty} \int_{C_{R}^{+}} z^{\beta} f(z) P_{m}\left(\frac{g_{\alpha}^{(1)}(z)}{2}\right) \mathrm{d} z=\lim _{N \rightarrow \infty} \int_{C_{R}^{+}} z^{\beta} f(z) \sum_{\nu=m+1}^{2 m+1} a_{\nu}\left(\frac{g_{\alpha}^{(1)}(z)}{2}\right)^{\nu} \mathrm{d} z=0
$$

which prove (15). Similarly, using again the same lemma, we get

$$
\lim _{N \rightarrow \infty} \int_{C_{R}^{-}} z^{\beta} f(z)\left(g_{\alpha}^{(2)}(z)\right)^{\nu} \mathrm{d} z=0
$$

and Formula (16) follows. 
Lemma 2. Let $n$ be an integer and $m$ a non-negative integer. We have

$$
\begin{aligned}
\psi_{\alpha, m}(z) & =1-2 P_{m}\left(\frac{g_{\alpha}^{(1)}(z)}{2}\right) \\
& =-1+2 P_{m}\left(\frac{g_{\alpha}^{(2)}(z)}{2}\right) .
\end{aligned}
$$

Proof. Note that, using $(11,12)$,

$$
\begin{aligned}
\phi_{\alpha}(z) & =1-g_{\alpha}^{(1)}(z) \\
& =-1+g_{\alpha}^{(2)}(z) .
\end{aligned}
$$

By applying the binomial theorem and integrating term by term, we get

$$
\psi_{\alpha, m}(z)=\frac{(2 m+1) !}{2^{2 m}(m !)^{2}} \int_{0}^{\phi_{\alpha}(z)}\left(1-t^{2}\right)^{m} \mathrm{~d} t .
$$

Replacing $\phi_{\alpha}(z)$ by the right-hand side of Formula (20), we obtain

$$
\begin{aligned}
\int_{0}^{\phi_{\alpha}(z)}\left(1-t^{2}\right)^{m} \mathrm{~d} t & =\int_{0}^{1}\left(1-t^{2}\right)^{m} \mathrm{~d} t+\int_{1}^{1-g_{\alpha}^{(1)}(z)}\left(1-t^{2}\right)^{m} \mathrm{~d} t \\
& =\frac{2^{2 m}(m !)^{2}}{(2 m+1) !}-2^{2 m+1} \int_{0}^{\frac{g_{\alpha}^{(1)}(z)}{2}} u^{m}(1-u)^{m} \mathrm{~d} u \\
& =\frac{2^{2 m}(m !)^{2}}{(2 m+1) !}-2^{2 m+1} \frac{(m !)^{2}}{(2 m+1) !} P_{m}\left(g_{\alpha}^{(1)}(z)\right) .
\end{aligned}
$$

So, in view of Formula (22), we get (18). Similarly, using (21), we obtain (19).

Lemma 3. Let $m$ be a non-negative integer and let $n$ be an integer. If $f$ is an entire function then we have

$$
\operatorname{Res}\left(z^{p} f(z) \psi_{\alpha, m}(z) ; z=\frac{j_{k}}{\tau}\right)=\frac{i(-1)^{n}(2 m+1) !}{2^{2 m}(m !)^{2} \tau^{p+1}} \sum_{\mu=0}^{m} \sum_{\nu=0}^{2 \mu} \frac{\left(\begin{array}{c}
m \\
\mu
\end{array}\right)\left(\begin{array}{c}
2 \mu \\
\nu
\end{array}\right)}{(2 \mu+1) ! \tau^{\nu}} a_{\mu, \nu}\left(j_{k}\right) f^{(\nu)}\left(\frac{j_{k}}{\tau}\right) .
$$

Proof. We have

$$
\begin{aligned}
\operatorname{Res}\left(z^{p} f(z) \psi_{\alpha, m}(z) ; z=\frac{j_{k}}{\tau}\right) & =\operatorname{Res}\left(z^{p} f(z) \frac{(2 m+1) !}{2^{2 m}(m !)^{2}} \sum_{\mu=0}^{m}\left(\begin{array}{c}
m \\
\mu
\end{array}\right) \frac{(-1)^{\mu}}{2 \mu+1}\left(i(-1)^{n} \frac{J_{-\alpha}(\tau z)}{J_{\alpha}(\tau z)}\right)^{2 \mu+1} ; z=\frac{j_{k}}{\tau}\right) \\
& =i(-1)^{n} \frac{(2 m+1) !}{2^{2 m}(m !)^{2}} \sum_{\mu=0}^{m}\left(\begin{array}{c}
m \\
\mu
\end{array}\right) \frac{1}{2 \mu+1} \operatorname{Res}\left(z^{p} f(z)\left(\frac{J_{-\alpha}(\tau z)}{J_{\alpha}(\tau z)}\right)^{2 \mu+1} ; z=\frac{j_{k}}{\tau}\right)
\end{aligned}
$$

which is equivalent to (23).

In the next lemma we consider, as above, the $a_{\mu, \nu}\left(j_{k}\right)$ defined by (5). 
Lemma 4. Let $p, m$ be non-negative integers. If $\mu$ and $\nu$ are non-negative integers such that $0 \leq \nu \leq 2 \mu$, $0 \leq \mu \leq m$ then there exists a constant $M$, independent of $k$, such that

$$
\left|a_{\mu, \nu}\left(j_{k}\right)\right| \leq M\left|j_{k}\right|^{p} \quad \text { for } \quad k= \pm 1, \pm 2, \ldots
$$

Proof. We note that

$$
J_{\alpha}^{(l)}\left(j_{k}\right)=O\left(1 /\left|j_{k}\right|^{1 / 2}\right), \quad l=1,2, \ldots
$$

This is indeed true for $l=1$ using the asymptotic relations (see p. 506 in [10])

$$
j_{k}=\left(k+\frac{\alpha}{2}-\frac{1}{4}\right) \pi+O\left(\frac{1}{k}\right)
$$

and the formula

$$
J_{\alpha}^{\prime}(z)=-\sqrt{\frac{2}{\pi z}} \sin \left(z-\frac{\alpha \pi}{2}-\frac{\pi}{4}\right)+O\left(\frac{1}{|z|^{\frac{3}{2}}}\right),|z| \rightarrow \infty,|\arg (z)|<\pi .
$$

For $l \geq 2$, we use use the principle of mathematical induction and the differential equation

$$
\begin{aligned}
z^{2} J_{\alpha}^{(l)}(z)+(2 l-3) z J_{\alpha}^{(l-1)}(z)+\left(z^{2}-\alpha^{2}+(l-2)^{2}\right) & J_{\alpha}^{(l-2)}(z) \\
& +2(l-2) z J_{\alpha}^{(l-3)}(z)+(l-2)(l-3) J_{\alpha}^{(l-4)}(z)=0,
\end{aligned}
$$

which is obtained by repeated differentiation of

$$
z^{2} J_{\alpha}^{\prime \prime}(z)+z J_{\alpha}^{\prime}(z)+\left(z^{2}-\alpha^{2}\right) J_{\alpha}(z)=0 .
$$

The same idea leads us to

$$
J_{-\alpha}^{(l)}\left(j_{k}\right)=O\left(1 /\left|j_{k}\right|^{1 / 2}\right), \quad l=1,2, \ldots
$$

Now, using Cauchy's integral formula, we may write

$$
a_{\mu, \nu}\left(j_{k}\right)=\frac{(2 \mu-\nu) !}{2 \pi} \int_{0}^{2 \pi} \frac{\left(j_{k}+\varepsilon \mathrm{e}^{\mathrm{i} \theta}\right)^{p}}{\left(\varepsilon \mathrm{e}^{\mathrm{i} \theta}\right)^{2 \mu-\nu}}\left(\frac{J_{-\alpha}\left(j_{k}+\varepsilon \mathrm{e}^{\mathrm{i} \theta}\right) \varepsilon \mathrm{e}^{\mathrm{i} \theta}}{J_{\alpha}\left(j_{k}+\varepsilon \mathrm{e}^{\mathrm{i} \theta}\right)}\right)^{2 \mu+1} \mathrm{~d} \theta
$$

where $\varepsilon$ is a small positive number. We have, using (28),

$$
\begin{aligned}
\left|J_{-\alpha}\left(j_{k}+\varepsilon \mathrm{e}^{\mathrm{i} \theta}\right)\right| & =\left|\sum_{l=0}^{\infty} J_{-\alpha}^{(l)}\left(j_{k}\right) \frac{\left(\varepsilon \mathrm{e}^{\mathrm{i} \theta}\right)^{l}}{l !}\right| \\
& \leq \sum_{l=0}^{\infty}\left|J_{-\alpha}^{(l)}\left(j_{k}\right)\right| \frac{\varepsilon^{l}}{l !}=O\left(\frac{1}{\left|j_{k}\right|^{1 / 2}}\right) .
\end{aligned}
$$


Also, using (24) and the inequality $\left|J_{\alpha}^{\prime}\left(j_{k}\right)\right|>c_{1} /\left|j_{k}\right|^{1 / 2}$,

$$
\begin{aligned}
\left|\frac{J_{\alpha}\left(j_{k}+\varepsilon \mathrm{e}^{\mathrm{i} \theta}\right)}{J_{\alpha}^{\prime}\left(j_{k}\right) \varepsilon \mathrm{e}^{\mathrm{i} \theta}}\right| & =\left|\sum_{l=0}^{\infty} \frac{J_{\alpha}^{(l+1)}\left(j_{k}\right)}{J_{\alpha}^{\prime}\left(j_{k}\right)} \frac{\left(\varepsilon \mathrm{e}^{\mathrm{i} \theta}\right)^{l}}{(l+1) !}\right| \\
& \geq 1-\sum_{l=1}^{\infty}\left|\frac{J_{\alpha}^{(l+1)}\left(j_{k}\right)}{J_{\alpha}^{\prime}\left(j_{k}\right)}\right| \frac{\varepsilon^{l}}{(l+1) !} \\
& \geq 1-c \sum_{l=1}^{\infty} \frac{\varepsilon^{l}}{(l+1) !}=1-c \frac{e^{\varepsilon}-\varepsilon-1}{\varepsilon},
\end{aligned}
$$

where $c$ is independent of $\varepsilon$. We infer that

$$
\left|\frac{J_{\alpha}\left(j_{k}+\varepsilon \mathrm{e}^{\mathrm{i} \theta}\right)}{\varepsilon \mathrm{e}^{\mathrm{i} \theta}}\right|>\frac{d}{\left|j_{k}\right|^{1 / 2}} \text { where } d \text { is independent of } \varepsilon \text {. }
$$

The conclusion of Lemma 4 follows from $(29,30,31)$.

Lemma 5. Let $m$ be a non-negative integer and let $\left\{\lambda_{k}\right\}, k= \pm 1, \pm 2, \ldots$ be an increasing sequence of real numbers such that $\lambda_{k+1}-\lambda_{k} \geq \delta>0$. If $f$ is entire and of exponential type such that

$$
\int_{-\infty}^{\infty}|f(x)| \mathrm{d} x<\infty
$$

then

$$
\sum_{\mu=0}^{m} \sum_{\nu=0}^{2 \mu} \sum_{\substack{k=-\infty \\ k \neq 0}}^{\infty}\left|f^{(\nu)}\left(\lambda_{k}\right)\right|<\infty
$$

Proof. Applying Theorem 11.3.3 of [4] to the functions $f(z)$ and $f(-z)$, we get

$$
\int_{0}^{\infty}\left|f^{(\nu)}(x)\right| \mathrm{d} x<\infty \text { and } \int_{0}^{\infty}\left|f^{(\nu)}(-x)\right| \mathrm{d} x<\infty
$$

Let $0 \leq \mu \leq m$ and $0 \leq \nu \leq 2 \mu$. Using Lemma 1 of $[6]$ with the function $f^{(\nu)}(z)$ and the sequence $\left\{\lambda_{k}\right\}_{k=1}^{\infty}$, we obtain

$$
\sum_{k=1}^{\infty}\left|f^{(\nu)}\left(\lambda_{k}\right)\right|<\infty
$$

Similarly, using Lemma 1 of [6] with the function $f^{(\nu)}(-z)$ and the sequence $\left\{-\lambda_{-k}\right\}_{k=1}^{\infty}$, we obtain

$$
\sum_{k=-\infty}^{-1}\left|f^{(\nu)}\left(\lambda_{k}\right)\right|=\sum_{k=1}^{\infty}\left|f^{(\nu)}\left(\lambda_{-k}\right)\right|<\infty .
$$

Since $(32,33)$ are valid for all $0 \leq \mu \leq m$ and $0 \leq \nu \leq 2 \mu$, we conclude that the lemma holds. 
We will use, in the proofs of Theorems 2 and 2', the following

Lemma 6. If $p=0,0 \leq \mu \leq m, 0 \leq \nu \leq 2 \mu$ and $f$ is an entire function of exponential type such that

$$
\int_{-\infty}^{\infty}|f(x)| \mathrm{d} x<\infty
$$

then

$$
\sum_{\substack{k=-\infty \\ k \neq 0}}^{\infty}\left|a_{\mu, \nu}\left(j_{k}\right) f^{(\nu)}\left(\frac{j_{k}}{\tau}\right)\right|<\infty
$$

Proof. Applying Lemma 4, we get

$$
\left|a_{\mu, \nu}\left(j_{k}\right) f^{(\nu)}\left(j_{k} / \tau\right)\right| \leq M\left|f^{(\nu)}\left(j_{k} / \tau\right)\right| .
$$

Recall that there is only a finite number of non-real zeros of $J_{\alpha}(z)$ (see Eq. (15.27) of [10]). So, in view of (25), there exists $K_{1}>0$ such that, for $k>K_{1}$,

$$
j_{k+1}-j_{k}>1 / 2 \text { for all } k>K_{1} .
$$

Hence, (34) follows using (35) and Lemma 5.

\section{Proofs of the theorems}

Proof of Theorems 1 and 1'. Observe that

$$
\left(\phi_{\alpha}(z)\right)^{2 \mu+1}=i(-1)^{\mu+n} z^{-(2 n+1)(2 \mu+1)}\left(\frac{G_{-\alpha}(\tau z)}{G_{\alpha}(\tau z)}\right)^{2 \mu+1}
$$

for $\mu=0,1, \ldots, m$. So we can write, in view of Formula (8),

$$
z^{p} \psi_{\alpha, m}(z) f(z)=z^{p} f(z) \frac{i(-1)^{n}(2 m+1) !}{2^{2 m}(m !)^{2}} \sum_{\mu=0}^{m}\left(\begin{array}{c}
m \\
\mu
\end{array}\right) \frac{1}{2 \mu+1} z^{-(2 n+1)(2 \mu+1)}\left(\frac{G_{-\alpha}(\tau z)}{G_{\alpha}(\tau z)}\right)^{2 \mu+1} .
$$

We infer that $z^{p} \psi_{\alpha, m}(z) f(z)$ is meromorphic on $\mathbb{C}$ with a possible pole at the point zero and poles at the points $j_{k} / \tau, k= \pm 1, \pm 2, \ldots$

On the other hand, Formula (25) implies that $\left|j_{N}\right|=\left|N \pi+\alpha \frac{\pi}{2}-\frac{\pi}{4}\right|+O\left(\frac{1}{N}\right)$. So, after a few calculations, we obtain

$$
\begin{aligned}
R^{2}-\left|\frac{j_{N}}{\tau}\right|^{2} & =\left(\frac{\pi}{\tau}\right)^{2}\left(N+\frac{\Re(\alpha)}{2}\right)-\left(\frac{\Im(\alpha) \pi}{2 \tau}\right)^{2}+O(1), \\
\left|\frac{j_{N+1}}{\tau}\right|^{2}-R^{2} & =\left(\frac{\pi}{\tau}\right)^{2}\left(N+\frac{\Re(\alpha)}{2}\right)+\frac{\pi^{2}}{2 \tau^{2}}+\left(\frac{\Im(\alpha) \pi}{2 \tau}\right)^{2}+O(1) .
\end{aligned}
$$

Hence, there exists $N_{0}>0$ such that

$$
\left|\frac{j_{N}}{\tau}\right|<R<\left|\frac{j_{N+1}}{\tau}\right| \quad \text { for } \quad N>N_{0} .
$$


Applying the residue theorem we find, for $N>N_{0}$,

$$
\int_{C_{R}} z^{p} \psi_{\alpha, m}(z) f(z) \mathrm{d} z=2 \pi i \sum_{\left|j_{k}\right|<R} \operatorname{Res}\left(z^{p} \psi_{\alpha, m}(z) f(z) ; z=\frac{j_{k}}{\tau}\right)+2 \pi i \operatorname{Res}\left(z^{p} \psi_{\alpha, m}(z) f(z) ; z=0\right) .
$$

(See the beginning of Section 4 for the definition of $C_{R}$.)

We divide the integral on the left-hand side of Formula (38) into two integrals, where the domain of integration of the first is on $C_{R}^{+}$and the second is on $C_{R}^{-}$. Then, using (18) in the first integral and (19) in the second, we find

$$
\begin{gathered}
\int_{C_{R}^{+}} z^{p} f(z) \mathrm{d} z-2 \int_{C_{R}^{+}} z^{p} f(z) P_{m}\left(\frac{g_{\alpha}^{(1)}(z)}{2}\right) \mathrm{d} z-\int_{C_{R}^{-}} z^{p} f(z) \mathrm{d} z+2 \int_{C_{R}^{-}} z^{p} f(z) P_{m}\left(\frac{g_{\alpha}^{(2)}(z)}{2}\right) \mathrm{d} z= \\
2 \pi i \sum_{\substack{k=-N \\
k \neq 0}}^{N} \operatorname{Res}\left(z^{p} \psi_{\alpha, m}(z) f(z) ; z=\frac{j_{k}}{\tau}\right)+2 \pi i \operatorname{Res}\left(z^{p} \psi_{\alpha, m}(z) f(z) ; z=0\right) .
\end{gathered}
$$

Since the function $z^{p} f(z)$ is entire we have, by Cauchy's theorem,

$$
\int_{C_{R}^{+}} z^{p} f(z) \mathrm{d} z=-\int_{-R}^{R} x^{p} f(x) \mathrm{d} x
$$

and

$$
\int_{C_{R}^{-}} z^{p} f(z) \mathrm{d} z=\int_{-R}^{R} x^{p} f(x) \mathrm{d} x
$$

Thus Formula (39) is equivalent to

$$
\begin{gathered}
\int_{-R}^{R} x^{p} f(x) \mathrm{d} x+\int_{C_{R}^{+}} z^{p} f(z) P_{m}\left(\frac{g_{\alpha}^{(1)}(z)}{2}\right) \mathrm{d} z-\int_{C_{R}^{-}} z^{p} f(z) P_{m}\left(\frac{g_{\alpha}^{(2)}(z)}{2}\right) \mathrm{d} z= \\
\quad-\pi i \sum_{\substack{k=-N \\
k \neq 0}}^{N} \operatorname{Res}\left(z^{p} \psi_{\alpha, m}(z) f(z) ; z=\frac{j_{k}}{\tau}\right)-\pi i \operatorname{Res}\left(z^{p} \psi_{\alpha, m}(z) f(z) ; z=0\right) .
\end{gathered}
$$

Taking the limit as $N \rightarrow \infty$ in $(42)$ and using $(15,16)$, we deduce that

$$
\int_{\rightarrow-\infty}^{\rightarrow \infty} x^{p} f(x) \mathrm{d} x=-\pi i \sum_{\substack{k=-\infty \\ k \neq 0}}^{\infty} \operatorname{Res}\left(z^{p} \psi_{\alpha, m}(z) f(z) ; z=\frac{j_{k}}{\tau}\right)-\pi i \operatorname{Res}\left(z^{p} \psi_{\alpha, m}(z) f(z) ; z=0\right) .
$$

Hence, Theorem 1' holds.

On the other hand, since

$$
\operatorname{Res}\left(z^{p} \psi_{\alpha, m}(z) f(z) ; z=0\right)=0
$$

whenever

$$
p-(2 n+1)(2 \mu+1) \geq 0 \text { for all } 0 \leq \mu \leq m,
$$


we conclude that Theorem 1 holds also.

Proof of Theorem 2 and 2'. Suppose first that $\tau=1$ and $p=0$. For $0 \leq \mu \leq m$ and $0 \leq \nu \leq 2 \mu$, let

$$
c_{\mu, \nu}:=\frac{(-1)^{n} \pi(2 m+1) !\left(\begin{array}{c}
m \\
\mu
\end{array}\right)\left(\begin{array}{c}
2 \mu \\
\nu
\end{array}\right)}{2^{2 m}(m !)^{2} \tau^{p+1}(2 \mu+1) ! \tau^{\nu}} a_{\mu, \nu}\left(j_{k}\right)
$$

and

$$
T_{\tau}\left(j_{k}\right):=c_{\mu, \nu} f^{(\nu)}\left(\frac{j_{k}}{\tau}\right) \text { for } k= \pm 1 \pm 2, \ldots
$$

According to the hypothesis, the function $f(z)$ satisfies the conditions of Theorem 1' with $\tau=1+\varepsilon$, where $\varepsilon$ is a given positive number. We therefore have

$$
\int_{-\infty}^{\infty} f(x) \mathrm{d} x=-\pi i \operatorname{Res}\left(\psi_{\alpha, m}(z) f(z) ; z=0\right)+\sum_{\substack{k=-\infty \\ k \neq 0}}^{\infty} \sum_{\mu=0}^{m} \sum_{\nu=0}^{2 \mu} T_{1+\varepsilon}\left(j_{k}\right) .
$$

Further, we show that for $\delta>0$ we can choose $K_{0}$ such that

$$
S_{\varepsilon}:=\sum_{\mu=0}^{m} \sum_{\nu=0}^{2 \mu}\left(\sum_{k=-\infty}^{-K_{0}}\left|T_{1+\varepsilon}\left(j_{k}\right)\right|+\sum_{k=K_{0}}^{\infty}\left|T_{1+\varepsilon}\left(j_{k}\right)\right|\right)<\frac{\delta}{3}
$$

if $\varepsilon \in[0,1 / 2]$.

By virtue of (25), we have for $k>K_{2}$ and $\varepsilon \in(0,1 / 2]$,

$$
\frac{1}{1+\varepsilon}\left(j_{k+1}-j_{k}\right)>\frac{1}{2}
$$

Let

$$
I_{k}:=\left[k-\frac{1}{2}, k+\frac{1}{2}\right]
$$

and let $\xi_{k}(k= \pm 1, \pm 2, \ldots)$ be a number in $I_{k}$ such that

$$
\left|f\left(\xi_{k}\right)\right|=\max _{x \in I_{k}}|f(x)| .
$$

The points $\xi_{k} \in[k-1 / 2, k)$ form a subsequence $\left\{\xi_{k}^{\prime}\right\}$ and those in $[k, k+1 / 2]$ form another subsequence $\left\{\xi_{k}^{\prime \prime}\right\}$. Note that $\xi_{k+1}^{\prime}-\xi_{k}^{\prime} \geq 1 / 2$ and that the same inequality is also valid if we replace $\xi_{k}^{\prime}$ by $\xi_{k}^{\prime \prime}$. So, by Lemma 5, we have

$$
\sum_{\mu=0}^{m} \sum_{\nu=0}^{2 \mu} \sum_{\substack{k=-\infty \\ k \neq 0}}^{\infty}\left|f^{(\nu)}\left(\xi_{k}^{\prime}\right)\right|<\infty \text { and } \sum_{\mu=0}^{m} \sum_{\nu=0}^{2 \mu} \sum_{\substack{k=-\infty \\ k \neq 0}}^{\infty}\left|f^{(\nu)}\left(\xi_{k}^{\prime \prime}\right)\right|<\infty
$$

whence

$$
\sum_{\mu=0}^{m} \sum_{\nu=0}^{2 \mu} \sum_{\substack{k=-\infty \\ k \neq 0}}^{\infty}\left|f^{(\nu)}\left(\xi_{k}\right)\right|<\infty
$$


On the other hand, using Lemma 6, we have

$$
\sum_{\mu=0}^{m} \sum_{\nu=0}^{2 \mu} \sum_{\substack{k=-\infty \\ k \neq 0}}^{\infty}\left|T_{1}\left(j_{k}\right)\right|<\infty
$$

Therefore, there exists $K_{0}>K_{2}$ such that

$$
\sum_{\mu=0}^{m} \sum_{\nu=0}^{2 \mu}\left(\sum_{k=-\infty}^{-\left[\frac{K_{0}}{2}\right]}\left|f^{(\nu)}\left(\xi_{k}\right)\right|+\sum_{k=\left[\frac{K_{0}}{2}\right]}^{\infty}\left|f^{(\nu)}\left(\xi_{k}\right)\right|\right)<\frac{\delta}{6 M_{2}}
$$

and

$$
\sum_{\mu=0}^{m} \sum_{\nu=0}^{2 \mu}\left(\sum_{k=-\infty}^{-K_{0}}\left|T_{1}\left(j_{k}\right)\right|+\sum_{k=K_{0}}^{\infty}\left|T_{1}\left(j_{k}\right)\right|\right)<\frac{\delta}{3}
$$

Using Lemma 4 with $p=0$, we see that there exists a constant $M_{1}$ such that $\left|a_{\mu, \nu}\left(j_{k}\right)\right| \leq M_{1}$ for all $k=$ $\pm 1, \pm 2, \ldots, 0 \leq \mu \leq m$ and $0 \leq \nu \leq 2 \mu$. So, there exists a positive constant $M_{2}$ such that $\left|c_{\mu, \nu}\left(j_{k}\right)\right| \leq M_{2}$. Therefore, we have

$$
S_{\varepsilon} \leq M_{2} \sum_{\mu=0}^{m} \sum_{\nu=0}^{2 \mu}\left(\sum_{k=-\infty}^{-K_{0}}\left|f^{(\nu)}\left(\frac{j_{k}}{1+\varepsilon}\right)\right|+\sum_{k=K_{0}}^{\infty}\left|f^{(\nu)}\left(\frac{j_{k}}{1+\varepsilon}\right)\right|\right) .
$$

Since, by (46), the intervals of the form $I_{k}$ contain at most two points of the sequence $\left\{j_{k} /(1+\varepsilon)\right\}, k=$ $\pm K_{2}, \pm\left(K_{2}+1\right), \ldots$, we have, in view of (49),

$$
\begin{aligned}
S_{\varepsilon} & \leq M_{2} \sum_{\mu=0}^{m} \sum_{\nu=0}^{2 \mu}\left(\sum_{k=-\infty}^{-K_{0}}\left|f^{(\nu)}\left(\frac{j_{k}}{1+\varepsilon}\right)\right|+\sum_{k=K_{0}}^{\infty}\left|f^{(\nu)}\left(\frac{j_{k}}{1+\varepsilon}\right)\right|\right) \\
& \leq 2 M_{2} \sum_{\mu=0}^{m} \sum_{\nu=0}^{2 \mu}\left(\sum_{k=\infty}^{-\left[\frac{K_{0}}{2}\right]}\left|f^{(\nu)}\left(\xi_{k}\right)\right|+\sum_{k=\left[\frac{K_{0}}{2}\right]}^{\infty}\left|f^{(\nu)}\left(\xi_{k}\right)\right|\right)<\frac{\delta}{3},
\end{aligned}
$$

which proves (45).

It is clear that, as $\varepsilon \rightarrow 0$, the function $f^{(\nu)}(z /(1+\varepsilon))$ converges uniformly on all compact subset of $\mathbb{C}$ to $f^{(\nu)}(z)(0 \leq \mu \leq m$ and $0 \leq \nu \leq 2 \mu)$. Therefore, there exists an $\varepsilon_{0}>0$ such that for $\varepsilon \in\left[0, \varepsilon_{0}\right]$,

$$
\sum_{\mu=0}^{m} \sum_{\nu=0}^{2 \mu}\left|\sum_{\substack{k=-K_{0}+1 \\ k \neq 0}}^{K_{0}-1} T_{1+\varepsilon}\left(j_{k}\right)-\sum_{\substack{k=-K_{0}+1 \\ k \neq 0}}^{K_{0}-1} T_{1}\left(j_{k}\right)\right|<\frac{\delta}{3}
$$


Thus, for $\varepsilon \in\left[0, \varepsilon_{0}\right]$, we have by virtue of (45) and the inequality (48),

$$
\begin{aligned}
\sum_{\mu=0}^{m} \sum_{\nu=0}^{2 \mu}\left|\sum_{\substack{k=-\infty \\
k \neq 0}}^{\infty} T_{1+\varepsilon}\left(j_{k}\right)-\sum_{\substack{k=-\infty \\
k \neq 0}}^{\infty} T_{1}\left(j_{k}\right)\right|< & \frac{\delta}{3}+\sum_{\mu=0}^{m} \sum_{\nu=0}^{2 \mu}\left(\sum_{k=-\infty}^{-K_{0}}\left|T_{1+\varepsilon}\left(j_{k}\right)\right|+\sum_{k=K_{0}}^{\infty}\left|T_{1+\varepsilon}\left(j_{k}\right)\right|\right) \\
& +\sum_{\mu=0}^{m} \sum_{\nu=0}^{2 \mu}\left(\sum_{k=-\infty}^{-K_{0}}\left|T_{1}\left(j_{k}\right)\right|+\sum_{k=K_{0}}^{\infty}\left|T_{1}\left(j_{k}\right)\right|\right)<\delta
\end{aligned}
$$

Hence,

$$
\lim _{\varepsilon \rightarrow 0} \sum_{\substack{k=-\infty \\ k \neq 0}}^{\infty} \sum_{\mu=0}^{m} \sum_{\nu=0}^{2 \mu} T_{1+\varepsilon}\left(j_{k}\right)=\sum_{\substack{k=-\infty \\ k \neq 0}}^{\infty} \sum_{\mu=0}^{m} \sum_{\nu=0}^{2 \mu} T_{1}\left(j_{k}\right)
$$

By letting $\varepsilon$ tend to zero in (44), we obtain

$$
\begin{aligned}
\int_{-\infty}^{\infty} f(x) \mathrm{d} x= & -\pi i \operatorname{Res}\left(\psi_{\alpha, m}(z) f(z) ; z=0\right) \\
& +\frac{(-1)^{n} \pi(2 m+1) !}{2^{2 m}(m !)^{2}} \sum_{\substack{k=-\infty \\
k \neq 0}}^{\infty} \sum_{\mu=0}^{m} \sum_{\nu=0}^{2 \mu} \frac{\left(\begin{array}{c}
m \\
\mu
\end{array}\right)\left(\begin{array}{c}
2 \mu \\
\nu
\end{array}\right)}{(2 \mu+1) !} a_{\mu, \nu}\left(j_{k}\right) f^{(\nu)}\left(j_{k}\right) .
\end{aligned}
$$

Remark that Formula (51) is equivalent to

$$
\begin{aligned}
\int_{-\infty}^{\infty} f(x) \mathrm{d} x= & -\pi i \operatorname{Res}\left(\psi_{\alpha, m}(z) f(z) ; z=0\right) \\
& +\frac{(-1)^{n} \pi(2 m+1) !}{2^{2 m}(m !)^{2}} \sum_{\substack{k=-\infty \\
k \neq 0}}^{\infty} \sum_{\mu=0}^{m} \frac{\left(\begin{array}{c}
m \\
\mu
\end{array}\right)}{2 \mu+1} \operatorname{Res}\left(f(z)\left(\frac{J_{-\alpha}(z)}{J_{\alpha}(z)}\right)^{2 \mu+1} ; z=j_{k}\right)
\end{aligned}
$$

which follows using the formula

$$
\sum_{\mu=0}^{m} \sum_{\nu=0}^{2 \mu} \frac{\left(\begin{array}{c}
m \\
\mu
\end{array}\right)\left(\begin{array}{c}
2 \mu \\
\nu
\end{array}\right)}{(2 \mu+1) !} a_{\mu, \nu}\left(j_{k}\right) f^{(\nu)}\left(j_{k}\right)=\sum_{\mu=0}^{m} \frac{\left(\begin{array}{c}
m \\
\mu
\end{array}\right)}{2 \mu+1} \operatorname{Res}\left(z^{p} f(z)\left(\frac{J_{-\alpha}(z)}{J_{\alpha}(z)}\right)^{2 \mu+1} ; z=j_{k}\right)
$$

with $p=0$. Note that Formula (53) is obtained using Lemma 3 and is valid for arbitrary $p$ and for $\tau=1$.

Replacing $f(z)$ by the function $z^{p} f(z)$ in Formula (52), we obtain

$$
\begin{aligned}
\int_{-\infty}^{\infty} x^{p} f(x) \mathrm{d} x= & -\pi i \operatorname{Res}\left(z^{p} \psi_{\alpha, m}(z) f(z) ; z=0\right) \\
& +\frac{(-1)^{n} \pi(2 m+1) !}{2^{2 m}(m !)^{2}} \sum_{\substack{k=-\infty \\
k \neq 0}}^{\infty} \sum_{\mu=0}^{m} \frac{\left(\begin{array}{c}
m \\
\mu
\end{array}\right)}{2 \mu+1} \operatorname{Res}\left(z^{p} f(z)\left(\frac{J_{-\alpha}(z)}{J_{\alpha}(z)}\right)^{2 \mu+1} ; z=j_{k}\right) .
\end{aligned}
$$


Now, applying (53), we infer that (54) is equivalent to (9). So, Theorem 2' holds.

Since

$$
\operatorname{Res}\left(z^{p} \psi_{\alpha, m}(z) f(z) ; z=0\right)=0
$$

whenever $n$ and $p$ satisfy (3), we conclude that Theorem 2 holds also.

\section{Concluding Remarks}

Using Formula (6), we get

$$
\begin{aligned}
a_{\mu, 2 \mu}\left(j_{k}\right)= & (-1)^{n}\left(\frac{2}{\pi}\right)^{2 \mu+1} \frac{j_{k}^{p-2 \mu-1}}{\left(J_{\alpha}^{\prime}\left(j_{k}\right)\right)^{4 \mu+2}} \\
a_{\mu, 2 \mu-1}\left(j_{k}\right)= & (-1)^{n}\left(\frac{2}{\pi}\right)^{2 \mu+1} \frac{j_{k}^{p-2 \mu-2}}{\left(J_{\alpha}^{\prime}\left(j_{k}\right)\right)^{4 \mu+2}}\left((p+\mu+1 / 2)+(2 \mu+1)\left(\frac{j_{k} J_{-\alpha}^{\prime}\left(j_{k}\right)}{J_{-\alpha}\left(j_{k}\right)}\right)\right) \\
a_{\mu, 2 \mu-2}\left(j_{k}\right)= & (-1)^{n}\left(\frac{2}{\pi}\right)^{2 \mu+1} \frac{j_{k}^{p-2 \mu-3}}{\left(J_{\alpha}^{\prime}\left(j_{k}\right)\right)^{4 \mu+2}}\left(2 \mu(2 \mu+1)\left(\frac{j_{k} J_{-\alpha}^{\prime}\left(j_{k}\right)}{J_{-\alpha}\left(j_{k}\right)}\right)^{2}\right. \\
& \left.+(2 \mu+1)(2 \mu+2 p)\left(\frac{j_{k} J_{-\alpha}^{\prime}\left(j_{k}\right)}{J_{-\alpha}\left(j_{k}\right)}\right)+p(2 \mu+p)+(2 \mu+1)\left(\frac{\mu}{2}+\frac{2}{3}\left(n^{2}+n\right)-\frac{2}{3} j_{k}^{2}\right)\right) .
\end{aligned}
$$

In order to obtain the above formulae, we use

$$
\begin{aligned}
\lim _{z \rightarrow j_{k}}\left(\frac{\left(z-j_{k}\right)}{J_{\alpha}(z)}\right)^{\prime} & =\frac{1}{J_{\alpha}^{\prime}\left(j_{k}\right)} \\
\lim _{z \rightarrow j_{k}}\left(\frac{\left(z-j_{k}\right)}{J_{\alpha}(z)}\right)^{\prime} & =\frac{1}{2 j_{k} J_{\alpha}^{\prime}\left(j_{k}\right)} \\
\lim _{z \rightarrow j_{k}}\left(\frac{\left(z-j_{k}\right)}{J_{\alpha}(z)}\right)^{\prime \prime} & =\frac{1}{6 J_{\alpha}^{\prime}\left(j_{k}\right)}\left(2-\frac{2 \alpha^{2}+1}{j_{k}^{2}}\right),
\end{aligned}
$$

which we obtain after a few calculations. We use also the relation

$$
J_{-\alpha}\left(j_{k}\right)=\frac{2(-1)^{n}}{\pi j_{k} J_{\alpha}^{\prime}\left(j_{k}\right)}
$$

which follows from the equation (3.12) of [10]

$$
J_{\alpha}^{\prime}(z) J_{-\alpha}(z)-J_{\alpha}(z) J_{-\alpha}^{\prime}(z)=\frac{2 \sin (\alpha \pi)}{\pi z} .
$$

In general, in order to compute more explicitly $a_{\mu, \nu}\left(j_{k}\right)$, we have to evaluate $J_{-\alpha}^{\prime}\left(j_{k}\right), J_{-\alpha}^{\prime \prime}\left(j_{k}\right), \ldots$ It is possible to evaluate $J_{-\alpha}^{\prime}\left(j_{k}\right)$ in terms of $J_{\alpha}^{\prime}\left(j_{k}\right)$. We use the formula

$$
J_{-\alpha}^{\prime}\left(j_{k}\right)=-J_{-\alpha+1}\left(j_{k}\right)-\frac{\alpha}{j_{k}} J_{-\alpha}\left(j_{k}\right)
$$


and we apply Lemma 1 of [1] with $p=2 n-1$ and $p=2 n$ to obtain the expression respectively of $J_{-\alpha+1}\left(j_{k}\right)$ and of $J_{-\alpha}\left(j_{k}\right)$ in terms of $J_{\alpha}^{\prime}\left(j_{k}\right)$ for $n \geq 0$. If $n$ is negative then we use Lemma 2 of [1] by taking $p=-2 n-1$ and $p=-2 n-2$ to get the expression respectively of $J_{-\alpha+1}\left(j_{k}\right)$ and of $J_{-\alpha}\left(j_{k}\right)$ in terms of $J_{\alpha}^{\prime}\left(j_{k}\right)$. The neat result is:

$$
J_{-\alpha}^{\prime}\left(j_{k}\right)= \begin{cases}(-1)^{n+1} \sum_{r=0}^{n} \frac{(2 n-2 r+1) !\left(\begin{array}{c}
n \\
r
\end{array}\right)\left(\begin{array}{c}
2 n-r \\
n
\end{array}\right)}{2^{2 n-2 r+1} j_{k}^{2 n-2 r+1}} J_{\alpha}^{\prime}\left(j_{k}\right) & \text { if } n \geq 0 \\
(-1)^{-n-1} \sum_{r=0}^{-n-1} \frac{(-2 n-2 r-1) !\left(\begin{array}{c}
-n-1 \\
r
\end{array}\right)\left(\begin{array}{c}
-2 n-r-2 \\
-n-1
\end{array}\right)}{2^{-2 n-2 r-1} j_{k}^{-2 n-2 r-1}} J_{\alpha}^{\prime}\left(j_{k}\right) & \text { if } n<0 .\end{cases}
$$

The expressions for $J_{-\alpha}^{\prime \prime}\left(j_{k}\right), J_{-\alpha}^{\prime \prime \prime}\left(j_{k}\right), \ldots$ in terms of $J_{\alpha}^{\prime}\left(j_{k}\right)$ follow from the differential equation (27) where we take $-\alpha$ instead of $\alpha$. Formulae $(14,19)$ of [1] give $\left(J_{\alpha}^{\prime}\left(j_{k}\right)\right)^{2}$ as a rational function of $j_{k}$, which is useful to compute the $a_{\mu, \nu}\left(j_{k}\right)$.

\section{REFERENCES}

[1] R. Ben Ghanem and C. Frappier, Spherical Bessel functions and explicit quadrature formula. Math. Comp. 66 (1997) $289-296$.

[2] R. Ben Ghanem, Quadrature formulae using zeros of Bessel functions as nodes. Math. Comp. 67 (1998) 323-336.

[3] R.P. Boas, Summation formulas and band-limited signals. Tôhoku Math. J. 24 (1972) 121-125.

[4] R.P. Boas, Entire functions. Academic Press, New York (1954).

[5] C. Frappier and P. Olivier, A quadrature formula involving zeros of Bessel functions. Math. Comp. 60 (1993) 303-316.

[6] G.R. Grozev and Q.I. Rahman, A quadrature formula with zeros of Bessel functions as nodes. Math. Comp. 64 (1995) $715-725$.

[7] R. Kress, On the general Hermite cardinal interpolation. Math. Comp. 26 (1972) 925-933.

[8] P. Olivier and Q.I. Rahman, Sur une formule de quadrature pour les fonctions entières. RAIRO Modél. Math. Anal. Numér. 20 (1986) 517-537.

[9] Q.I. Rahman and G. Schmeisser, Quadrature formulae and functions of exponential type. Math. Comp. 54 (1990) $245-270$.

[10] G.N. Watson, A treatise on the theory of Bessel functions, 2nd edn. Cambridge University Press, Cambridge (1966). 